\title{
Caracterización experimental y numérica de la articulación del hombro en la técnica del estilo de crol en natación
}

\author{
Marcos Javier Hernández Gill ${ }^{1}$, Jorge Grasa Orús², Jose Javier Marín Zurdo \\ ${ }^{1}$ Becado en el I3A (AMB + IDERGO). \\ Instituto de Investigación en Ingeniería de Aragón (I3A) \\ Universidad de Zaragoza, Mariano Esquillor s/n, 50018, Zaragoza, Spain. \\ Tel. +34-976762707, e-mail: marcoshndez@gmail.com \\ ${ }^{2}$ Applied Mechanics and Bioengineering (AMB). \\ ${ }^{3}$ IDERGO.
}

\section{Resumen}

Con ayuda de los sistemas y marcadores ópticos es posible caracterizar el movimiento tridimensional, en este caso, de la articulación del hombro durante la brazada de crol en un laboratorio fuera del agua, para posteriormente simular su comportamiento biomecánico utilizando el método de los elementos finitos.

\section{Introducción}

La natación es un deporte donde dadas las grandes ventajas que ofrece ha ido obteniendo un mayor protagonismo ya no sólo a nivel terapéutico sino también, gracias al avance de la tecnología, a nivel competitivo tal y como queda demostrado en el número de estudios publicados (Heinlein y Cosgarea, 2010).

Un nadador competitivo, de media, puede llegar a nadar entre $60-80 \mathrm{~km} / \mathrm{semana}$ con valores en rango de 8-10 brazadas por cada $25 \mathrm{~m}$, lo que sugiere que el número de brazadas que puede dar un nadador a la semana asciende a un total aproximado de 30.000 a la semana, con cada brazo (Heinlein y Cosgarea, 2010), donde la mayor parte de la fuerza propulsiva proviene de los brazos (Morouço et al., 2015).

No es de extrañar, que las lesiones más comunes en natación se focalicen en la articulación del hombro (Abgarov et al., 2012, Khodaee et al., 2016) junto a cadera y rodillas provocadas por movimientos cíclicos; así mismo en el cuello y lumbar por postura antinatural o forzada, dado que la morfología del ser humano no está preparada para desplazarse en el medio acuático (Llana Belloch et al., 2011).

Este artículo se puede subdividir en dos partes diferenciadas, en la primera se estudiarán experimentalmente, las fases de la brazada del estilo de Crol, con la peculiaridad de ser tratado bajo condiciones de laboratorio y fuera del agua, diferenciando, a priori, entre dos técnicas. La segunda parte partirá de los resultados obtenidos del capítulo anterior, para su implementación en un modelo de elementos finitos, formado por el húmero y el deltoides.

\section{Caracterización experimental}

A partir de un sistema óptico de captura de movimiento (MoCap) basado en agrupación de marcadores que se fijan en los segmentos corporales a monitorizar, se ha caracterizado la biomecánica de la articulación del hombro bajo condiciones de laboratorio, de siete (7) sujetos entrenados y federados en activo en categoría absoluta en la presente temporada 2018 - 2019 por la comunidad autónoma de Aragón, particularmente de la provincia de Zaragoza, cuya condición previa es haber participado en algún campeonato de España.

La prueba consiste en la simulación del nado de la técnica de crol en un laboratorio para dos tipos de técnica diferentes (Doblando codos y Molinillo), donde el nadador, con los marcadores colocados, se sitúa sobre un banco en el que se ha colocado una espuma de densidad calibrada donde puede apoyar la zona pélvica y de ese modo simular la acción de nado.

Varios autores aceptan las fases propuestas por Chollet et al., 2000, donde se distinguen y describen cuatro fases del nado como son (Phase A: Entry and Catch, Phase B: Pull, Phase C: Push, Phase D: Recovery).

En este estudio se ha determinado que sería conveniente dividir y diferenciar entre cinco fases, como son; agarre (Catch), tirón (Pull), empuje (Push), recobro (Recovery) y deslizamiento (Glide), caracterizados por seis instantes. (Ver figura 1 y 2)

Adicionalmente a las variables ya estudiados en la literatura, hemos querido proponer una serie de 
parámetros que permiten obtener más información sobre la forma de nadar del sujeto. Entre ellos, los parámetros propuestos son: Rolido, Angulo del Recobro, Profundidad de la mano, Altura de la Mano, Diferencia Mano-Codo, Anchura Brazada (Ver figura 3).

\section{Simulación numérica de la articulación}

Con los resultados experimentales de la caracterización del movimiento de la articulación del hombro, se ha implementado un modelo de elementos finitos en un software comercial (Abaqus), con el fin de conocer las tensiones y deformaciones a las que se ve afectados los músculos que componen dicha articulación.

Los modelos 3D (Húmero y Acromial (parte del deltoides)) provienen de una base de datos desarrollada por Mitsuhashi et al 2008 accesible para la descarga de geometrías reales.

En cuanto a las características y comportamiento mecánico, se ha considerado el hueso como un sólido rígido, dado que sus deformaciones frente a la de los músculos es despreciable, mientras que, una primera aproximación considerará los músculos como un material isótropo e hiperelástico (Mooney-Rivlin), esto permitirá conocer su comportamiento pasivo.

Las condiciones de contorno que afectan al hueso, son las de un conector tipo Cardan (ZXY), cuyas amplitudes serán las obtenidas de la caracterización. (Ver figura 4)

\section{Conclusiones}

El rolido o roll tiene una función fundamental, no solo a nivel de gasto energético o alineación del cuerpo, sino también para evitar lesiones en los hombros. El ángulo formado por el hombro, durante el instante de riesgo era superior en el lado predominante (derecho) lo que implica una mayor perpendicularidad, entre el tronco y el hombro, además se refuerza con una menor rotación longitudinal del cuerpo. Cuando existe ese rolido, el ángulo de riesgo disminuye notablemente. Siendo la serie 2 más perjudicial que la serie 1. (Ver figura 3, Max.Roll $\left[{ }^{\circ}\right]$ y Min.Roll $\left.\left[\left[^{\circ}\right]\right]\right)$.
El conjunto de este artículo, permite en un futuro cercano, realizar un estudio de valoración funcional y lesiones individualizado, donde a partir de la reconstrucción de la geometría del tejido duro y blando y sus características mecánicas (individuales) de un sujeto obtenida mediante tomografía computerizada, de un estudio de caracterización del movimiento (MoCap ópticos o inerciales) y su implementación en un software de elementos finitos, será posible particularizar el análisis a las condiciones específicas de individuo; tendencia que irá al alza en la prevención de lesiones en el ámbito deportivo.

\section{REFERENCIAS}

ABGAROV, A., FRASER-THOMAS, J. y BAKER, J., 2012. Understanding trends and risk factors of swimmingrelated injuries in varsity swimmers. Clinical Kinesiology, ISSN 08969620.

CHOLLET, D., CHALIES, S. y CHATARD, J.C., 2000. A new index of coordination for the crawl: Description and usefulness. International Journal of Sports Medicine, ISSN 01724622. DOI 10.1055/s-2000-8855.

HEINLEIN, S.A. y COSGAREA, A.J., 2010. Biomechanical considerations in the competitive swimmer's shoulder. 2010. S.l.: s.n.

KHODAEE, M., EDELMAN, G.T., SPITTLER, J., WILBER, R., KRABAK, B.J., SOLOMON, D., RIEWALD, S., KENDIG, A., BORGELT, L.M., RIEDERER, M., PUZOVIC, V. y RODEO, S., 2016. Medical Care for Swimmers. 2016. S.l.: s.n.

LLANA BELLOCH, S., PÉREZ SORIANO, P. y APARICIO APARICIO, I., 2011. Historia de la Natación I: desde la Prehistoria hasta la Edad Media. Citius, altius, fortius: humanismo, sociedad y deporte: investigaciones $y$ ensayos, ISSN 1888-6744.

MITSUHASHI N, FUJIEDA K, TAMURA T, KAWAMOTO S, TAKAGI T, OKUBO K. BodyParts3D: 3D structure database for anatomical concepts. Nucleic Acids Res. 2008;37(Database doi:10.1093/nar/gkn613 issue):D782-D785.

MOROUÇO, P.G., MARINHO, D.A., IZQUIERDO, M., NEIVA, H. y MARQUES, M.C., 2015. Relative Contribution of Arms and Legs in 30 s Fully Tethered Front Crawl Swimming. BioMed Research International, ISSN 23146141. DOI 10.1155/2015/563206. 


\section{Anexo. Figuras y tablas}
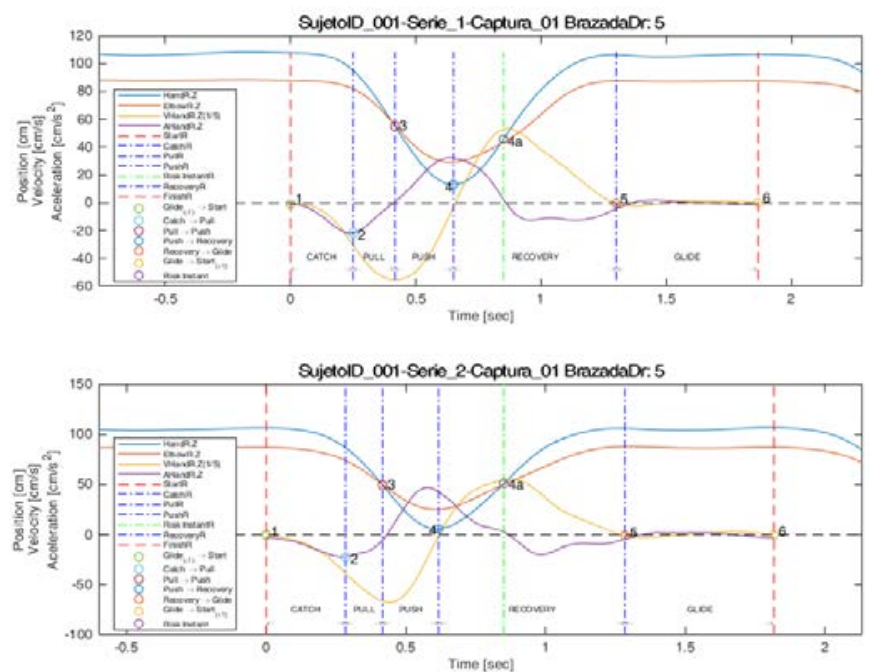

Fig. 1. (a) Representación gráfica de los eventos de la brazada derecha número cinco (5) del SujetoID001 - Serie 1 - Captura 01. (b) representación gráfica de los eventos de la brazada derecha número cinco (5) del SujetoID001 - Serie 2 - Captura 01. En azul, se representa la ManoDr.Z, naranja el CodoDr.Z, en amarillo, la velocidad de la ManoDr.Z, en morado la aceleración de la ManoDr.Z. Las rectas verticales, corresponde a los instantes de tiempo de los puntos: (1) Catch, (2) Pull, (3) Push, (4) Recovery, (4a) Instante de Riesgo, (5) Glide, (6) Catch+1.
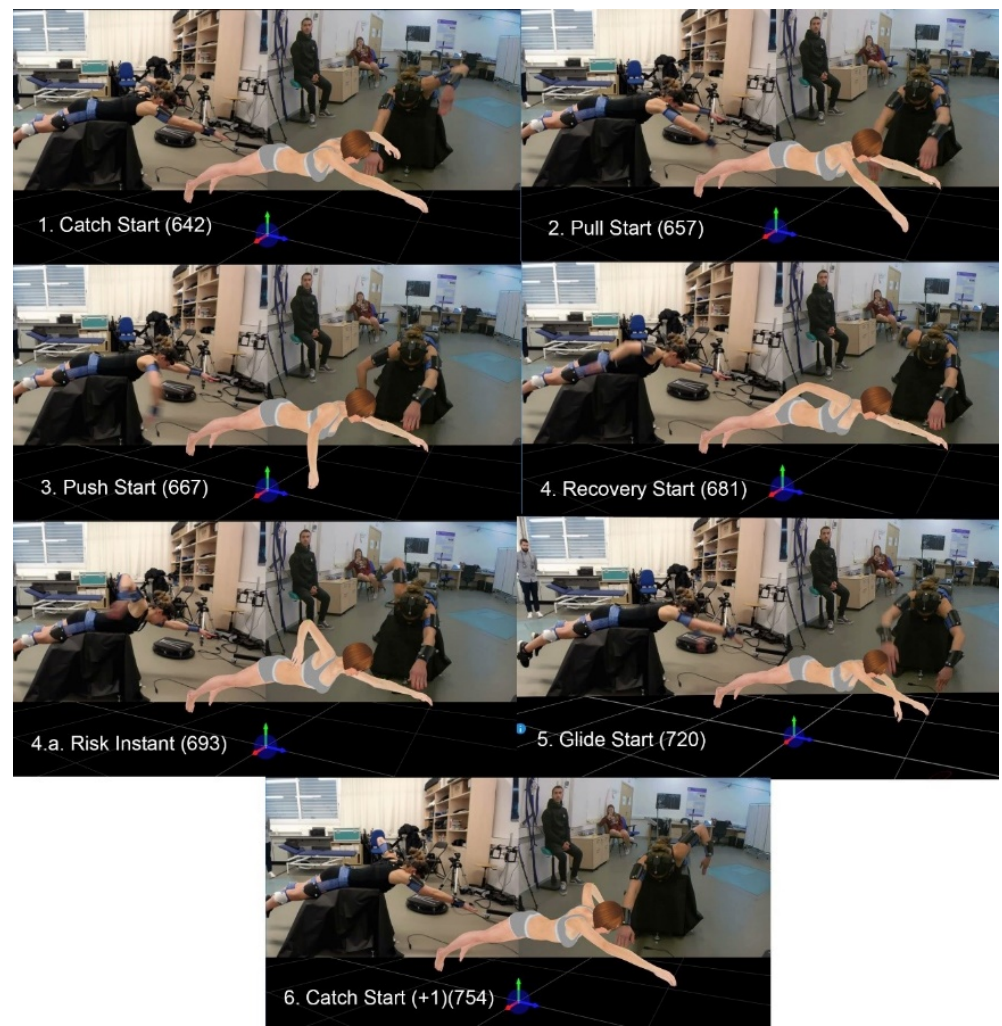

Fig. 2. Ensayo experimental MoCap de los eventos de la brazada derecha número cinco (5) del SujetoID001 - Serie 1 - Captura 01. Con correspondencia a la figura 2.a. siendo (1) Catch, (2) Pull, (3) Push, (4) Recovery, (4a) Instante de Riesgo, (5) Glide, (6) Catch+1 (entre paréntesis el frame correspondiente en el video). 


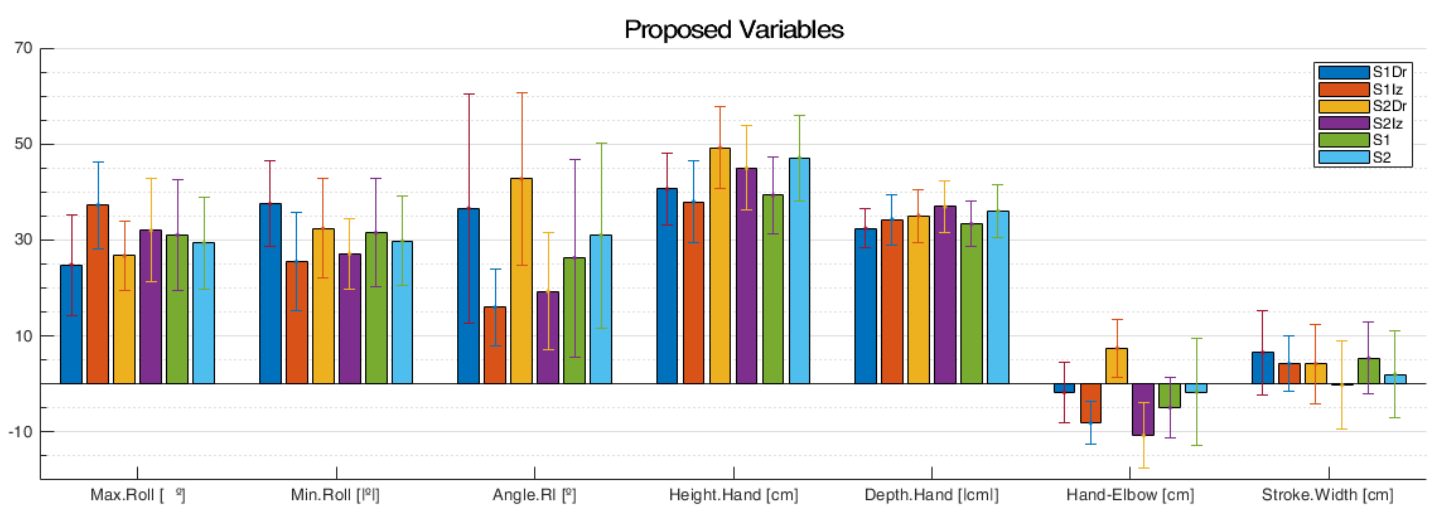

Fig. 3. Media y desviación típica de las variables propuestas durante el artículo diferenciando entre lados [Dr/Iz] y Series [S1/S2].

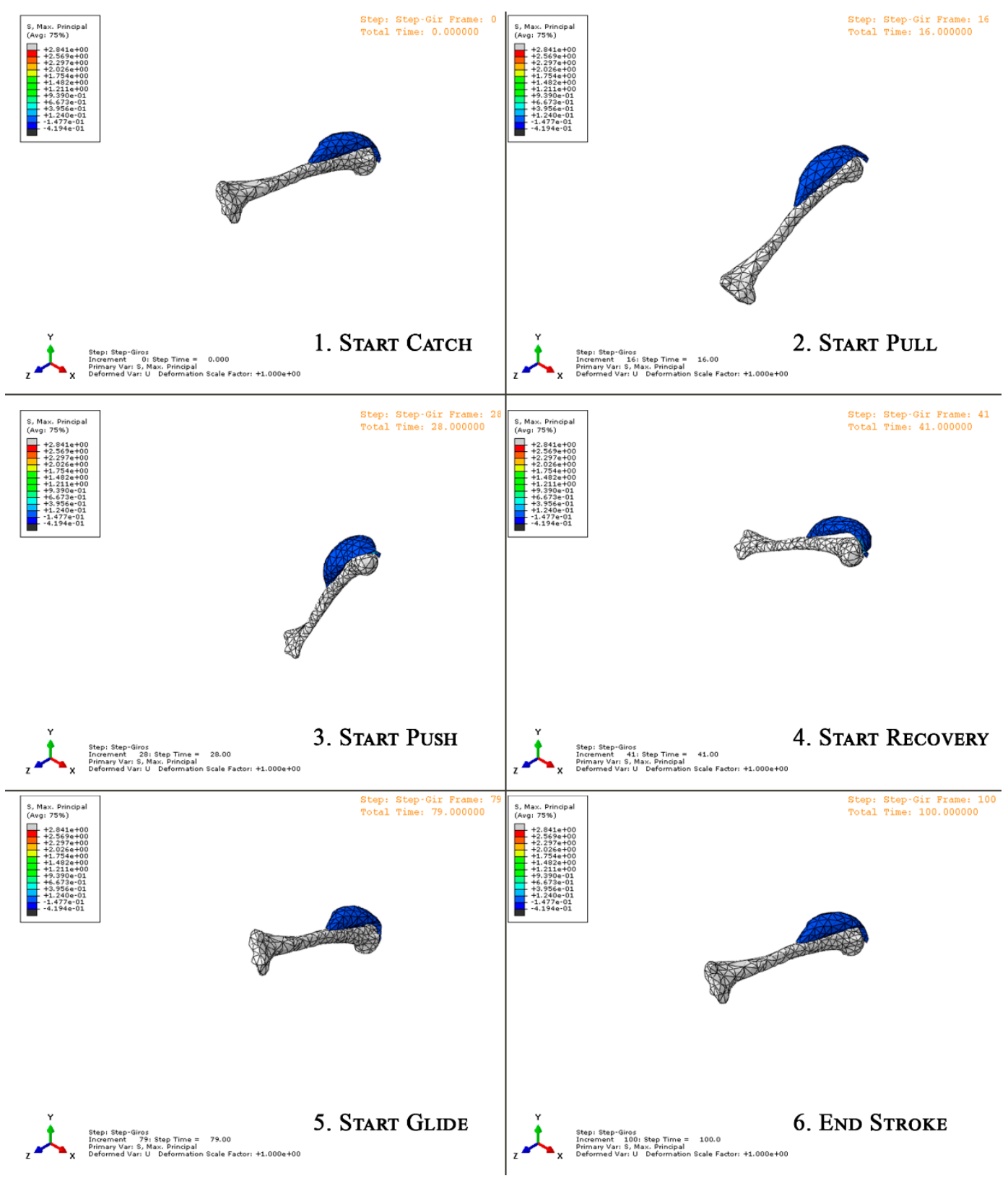

Fig. 4. Resultados computacionales del modelo del hombro, formado por el húmero y el Acromial (parte del deltoides) implementado en Abaqus

Revista “Jornada de Jóvenes Investigadores del I3A”, vol. 7 (Actas de la VIII Jornada de Jóvenes Investigadores del I3A - 6 de junio de 2019). ISSN 2341-4790. 